\title{
Observation of Anisotropic Charge Density Wave in Layered 1T-TiSe 2
}

Qiao Qiao ${ }^{1,2}$, Songsong Zhou ${ }^{3}$, Jing Tao ${ }^{2}$, Jin-Cheng Zheng ${ }^{4}$, Lijun $\mathrm{Wu}^{2}$, Samuel T. Ciocys ${ }^{5}$, Maria Iavarone ${ }^{1}$, David J. Srolovitz ${ }^{3,6}$, Goran Karapetrov ${ }^{5}$, and Yimei Zhu ${ }^{2}$

${ }^{1 .}$ Department of Physics, Temple University, Philadelphia, Pennsylvania 19122, USA

2. Condensed Matter Physics and Materials Science Department, Brookhaven National Laboratory, Upton, New York 11973, USA

3. Department of Materials Science and Engineering, University of Pennsylvania, Philadelphia, Pennsylvania 19104, USA

4. Department of Physics, and Collaborative Innovation Center for Optoelectronic Semiconductors and Efficient Devices, Xiamen University, Xiamen, Fujian 361005, China

5. Department of Physics, Drexel University, Philadelphia, Pennsylvania 19104, USA

6. Department of Mechanical Engineering and Applied Mechanics, University of Pennsylvania, Philadelphia, Pennsylvania 19104, USA

Material systems containing charge density waves (CDWs) are ideal for studying structural symmetry breaking induced by electron modulation that would give rise to unconventional macroscopic phenomena such as high- $\mathrm{T}_{\mathrm{c}}$ superconductivity and colossal magnetoresistance. Layered $1 T-\mathrm{TiSe}_{2}$ has attracted particular research interests given its rich phase diagram consisting of commensurate and incommensurate CDWs, as well as superconductivity. These phases can be tuned by temperature, pressure [1], electric field [2], or intercalation [3]. Moreover, superconductivity is found to be strongly connected to the formation of CDW domain walls [1-2,4]. In this study, we conduct a full-scale study on $1 T-\mathrm{TiSe}_{2}$ using an integration of techniques to investigate, at cryogenic temperatures, the real space, reciprocal space, and energy space properties [5]. Our results show that the CDW forms threedimensional cylinder shape domain, with different in-plane and out-of-plane coherence lengths. First principles density functional theory (DFT) calculations are integrated to explore the electronic feature of CDW in $1 \mathrm{~T}-\mathrm{TiSe}_{2}$.

Figure 1 shows the comparison between NED patterns taken before (figure 1a) and after (figure 1b) insitu liquid nitrogen cooling. At $89 \mathrm{~K}$, we find clear evidence of $2 \times 2 \times 2$ superlattice between the zero order Laue zone (ZOLZ) and the first order Laue zone (FOLZ), which is generated by the commensurate CDW phase. The Bragg peaks in the NED pattern can be classified into several categories (figure 1c) that provide information of in-plane or out-of-plane properties, from either the fundamental lattice or the CDW superlattice. By quantitatively analyzing the NED pattern, we derived that in $1 \mathrm{~T}-\mathrm{TiSe}_{2}$, the CDW domains form cylinder shape, their in-plane coherence length is short, but out-of-plane coherence length is long.

In-situ scanning CBED at liquid helium temperature is then utilized to perform a three-dimensional investigation. Figure $2 \mathrm{a}$ is a mapping of the CDW signal intensity, showing stark contrast between the CDW maxima (figure 2b) and minima (figure 2c), and the CDW maxima form a domain of $\sim 10 \mathrm{~nm}$. Within this domain, the CDW signal is strong and uniform, indicating that the long-range coherence of the out-of-plane component is preserved. Position averaged CBED was used to accurately measure sample thickness, which was found to be $17.5 \mathrm{~nm}$. Combining scanning CBED mapping with the NED pattern, we conclude that a typical CDW domain forms a cylinder shape, with in-plane coherence length of $\sim 10 \mathrm{~nm}$ and out-of-plane coherence length with $17.5 \mathrm{~nm}$ lower bound. 
Supported by DFT calculation, EELS can confirm the CDW state with high spatial resolution, and serve as a step toward characterization of electronic structure change at domain boundaries. Our integrated approach to investigate the three-dimensional nature of CDW domain can be further used to correlate, for example, CDW and superconductivity, as well as to understand emerging behavior in thin films due to the reduced dimensionality [6].

\section{References:}

[1] YI Joe et al, Nature Physics 10 (2014), p. 421.

[2] LJ Li et al, Nature $\mathbf{5 2 9}$ (2016), p. 185.

[3] E Morosan et al, Nature Physics 2 (2006), p. 544.

[4] S Yan et al, Physical Review Letters 118 (2017), p. 106405.

[5] Q Qiao et al, Physical Review Materials 1 (2017), p. 054002.

[6] This work was supported as part of the CCDM, and EFRC funded by the U.S. DOE BES under Grant Number DE-SC0012575. J.T., L.W., and Y.Z. were supported by the Materials Science and Engineering Division, U.S. DOE BES, under Grant Number DE-SC0012704.
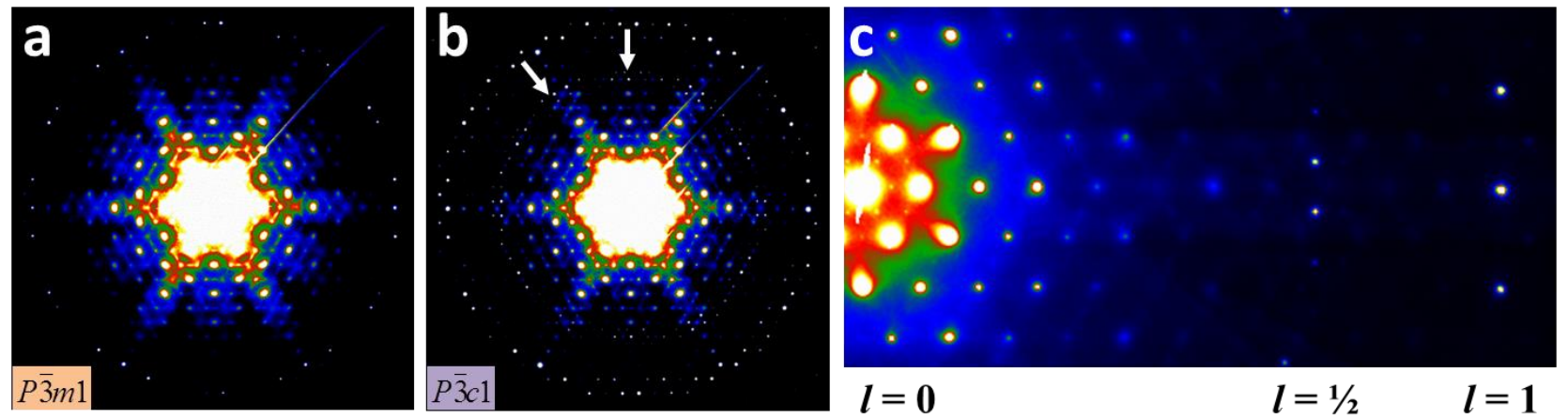

Figure 1. (a) NED pattern acquired at room temperature showing the $P-3 m 1$ space group. (b) NED pattern acquired at $89 \mathrm{~K}$ showing the $P-3 c 1$ space group, with $(1 / 21 / 21 / 2)$ Bragg peaks emerge between ZOLZ and FOLZ. (c) NED pattern cropped from (b) with reduced intensity saturation of the ZOLZ, showing Bragg peaks corresponding to the superlattice in ZOLZ $(l=0)$ and the CDW ring $(l=1 / 2)$.
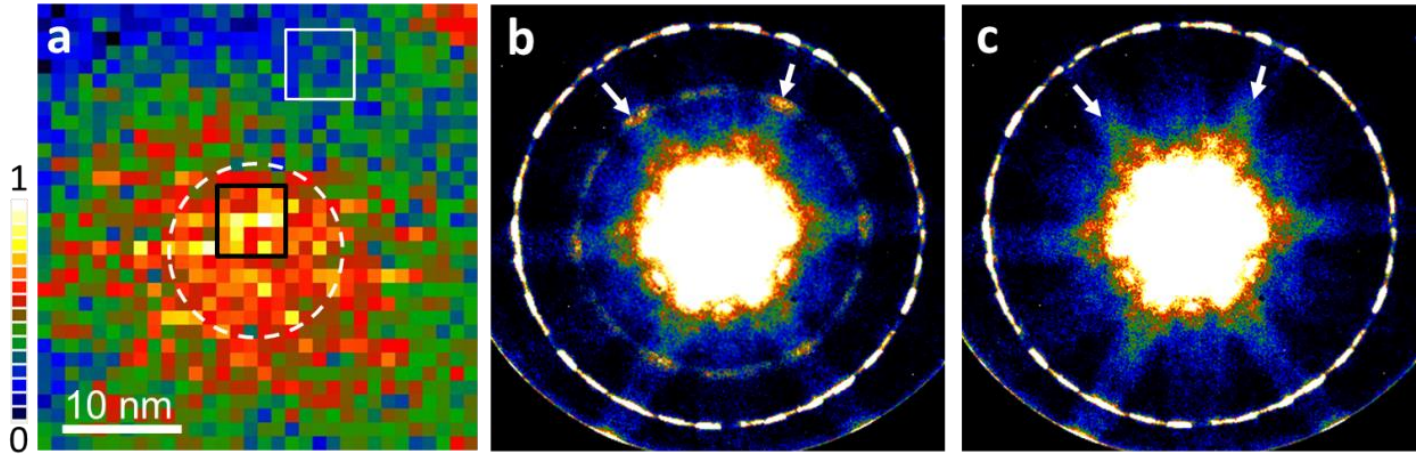

Figure 2. (a) Real space mapping of the CDW line intensity, showing domain structure of $\sim 10 \mathrm{~nm}$. CBED patterns are extracted from the black and white squares, as shown in (b) and (c), respectively. (b) CBED pattern showing visible CDW line, indicating $2 \times 2 \times 2$ superlattice in this area; (c) CBED patterns showing no CDW line, indicating the original $1 \times 1 \times 1$ structure in this area. 